\title{
STRONG HOMOTOPY EQUIVALENCE OF 3-MANIFOLDS
}

\author{
BY D. R. MCMILLAN, JR. ${ }^{1}$
}

\author{
Communicated by R. H. Bing, May 8, 1967
}

1. Introduction. Let $M$ be a topological space and let $X$ be a compact subset of $M$. After [2], we say that $X$ has property $U V^{\infty}$ (or " $X \in U V^{\infty}$ ") in $M$ if for each open set $U \subset M$ such that $X \subset U$, there is an open set $V$ such that $X \subset V \subset U$ and $V$ is contractible to a point in $U$. It is known [2] that each finite-dimensional compact absolute retract (i.e., retract of a cell) has property $U V^{\infty}$ under some embedding in Euclidean space, and that if one embedding of a compact set $X$ in a manifold has property $U V^{\infty}$, then so does every embedding of $X$ in a manifold.

Armentrout has shown ( $\$ 10$ of [2]) that if $M^{n}$ and $N^{n}$ are closed (i.e., compact and boundaryless) topological $n$-manifolds, and if $f$ is a continuous mapping of $M^{n}$ onto $N^{n}$ such that $f^{-1}(y) \in U V^{\infty}$ for each $y \in N^{n}$, then $f$ is a homotopy equivalence. We shall call such a mapping a strong homotopy equivalence of $M^{n}$ onto $N^{n}$. If $n=3$ and if $f$ is cellular (i.e., each set $f^{-1}(y)$ is cellular-and hence $U V^{\infty}$ ), then he has shown [1] that $M^{3}$ and $N^{3}$ are homeomorphic. It is our purpose here to note that if there is a strong homotopy equivalence of $M^{3}$ on to $N^{3}$, then $M^{3}$ and $N^{3}$ differ by only a finite number of homotopy 3 -cells (Corollary 2.1). Hence, modulo the Poincare conjecture, $M^{3}$ and $N^{3}$ are homeomorphic. If there is also a strong homotopy equivalence of $N^{3}$ onto $M^{3}$, then $M^{3}$ and $N^{3}$ are homeomorphic (independently of the Poincaré conjecture).

If $X$ is a compact subset of the interior of a piecewise-linear $n$ manifold $M^{n}, n \geqq 3$, and if $X \in U V^{\infty}$, then we shall say that $X$ satisfies the cellularity criterion in $M^{n}$ if for each open set $U \subset M^{n}$ such that $X \subset U$, there is an open set $V$ such that $X \subset V \subset U$ and each loop in $V-X$ is contractible in $U-X$. If $n \geqq 5$ and $X \in U V^{\infty}$, then $X$ is cellular (with respect to piecewise-linear cells) in $M^{n}$ if and only if the cellularity criterion holds (see [5]). For the situation in the 3dimensional case, see Theorem 1.

We shall use $E^{n}$ and $S^{n}$ to denote Euclidean $n$-space, and the $n$-sphere, respectively. The term "manifold" applies only to a connected space, unless stated otherwise. If $G$ is a disjoint collection of closed subsets of a space $X$ such that the union of the elements of $G$ is

\footnotetext{
1 Alfred P. Sloan Fellow.
} 
$X$, we shall say that $G$ is an upper semicontinuous decomposition of $X$ if for each $g \in G$ and for each open set $U \subset X$ such that $g \subset U$, there is an open set $V$ such that $g \subset V \subset U$ and such that each element of $G$ which intersects $V$ is contained in $U$.

2. Cellularity of inverse sets. The following is an improved version of Theorem $1^{\prime}$ of [5].

THEOREM 1. Let $M^{3}$ be a piecewise-linear 3-manifold without boundary and let $X$ be a compact subset of $M^{3}$ such that $X \in U V^{\infty}$ and $X$ satisfies the cellularity criterion in $M^{3}$. Then, for each open set $U \subset M^{3}$ such that $X \subset U$, there is a compact, polyhedral, contractible 3-manifold $H$ such that

$$
X \subset \text { Int } H \subset H \subset U,
$$

and such that $H-X$ is topologically $S^{2} \times[0,1)$.

Proof. We assume a familiarity with Lemma 1 of [5]. Let an arbitrary open set $V \subset M^{3}$ be given such that $X \subset V$. The first step is to show that there is a compact 3-manifold-with-boundary $H \subset V$ such that $X \subset$ Int $H$ and such that $H$ is a "homotopy cube-with-handles," that is, $H$ is obtained from a homotopy 3 -cell by adding orientable handles of index one to its boundary.

To do this, we make several applications of the $U V^{\infty}$ property and of regular neighborhoods to find compact 3-manifolds-with-boundary $K$ and $M_{1}$ ( $K$ may not be connected) such that

$$
X \subset \operatorname{Int} K \subset K \subset \text { Int } M_{1} \subset M_{1} \subset V,
$$

such that $M_{1}$ is contractible in $V$ and $\mathrm{Bd} M_{1}$ is connected and nonempty, and such that each loop in $K$ is contractible in $M_{1}$. Note that each polyhedral 2-sphere in $M_{1}$ bounds a homotopy 3-cell in $V$ and, since $\mathrm{Bd} M_{1}$ is connected, this homotopy 3-cell lies in $\mathrm{M}_{1}$. We are now in a position to repeat, using the same notation, the argument outlining the proof of Lemma 1 in [5]. The only difference is that in the present situation we know only that a polyhedral 2-sphere in $M_{1}$ bounds a homotopy 3-cell in $M_{1}$, rather than a piecewise-linear 3-cell. This will complete the first step of the proof, and makes use only of the fact that $X \in U V^{\infty}$.

The second step is to show, using the fact that $X$ satisfies the cellularity criterion, that $H$ can be chosen to be a homotopy 3-cell. For this we appeal to the proof of Theorem $1^{\prime}$ of [5], to "cut the handles" of 
our homotopy 3-cell-with-handles, without deleting any part of $X$ in the process.

By the first two steps, we may find homotopy 3-cells $H_{1}, H_{2}, \cdots$, such that $H_{i+1} \subset$ Int $H_{i}$ and $X=\bigcap_{i=1}^{\infty} H_{i}$. The third step, which will complete the proof, is to show that there exists an integer $N$ such that $A_{i}=H_{i}-\operatorname{Int} H_{i+1}$ is topologically $S^{2} \times[0,1]$ (a "3-annulus") for each $i>N$.

Let $F_{i} \subset$ Int $A_{i}$ be a polyhedral homotopy 3-cell obtained from $A_{i}$ by tunneling along an arc from one component of $\mathrm{Bd} A_{i}$ to the other, and then shaving off a product neighborhood of the boundary of the resulting 3-manifold. Then $F_{i}$ is a 3-cell if and only if $A_{i}$ is a 3-annulus. A result of Kneser (pp. 252-255 of [3]) implies that, since $H_{1}$ is compact, there is an integer $N$ such that $H_{1}$ does not contain more than $N$ disjoint, polyhedral homotopy 3-cells which fail to be 3-cells (apply Kneser's result to the "double" of $H_{1}$ ). This is the required $N$. The theorem follows.

Corollary 1.1. Assume the hypotheses of Theorem 1, and let $G$ be the upper semicontinuous decomposition of $M^{3}$ whose only nondegenerate element is $X$. Then the decomposition space $M^{3} / G$ is a 3-manifold which can be obtained from $M^{3}$ by removing a compact, polyhedral homotopy 3-cell and replacing it by a piecewise-linear 3-cell.

Theorem 2. Suppose that $M^{3}$ and $N^{3}$ are closed piecervise-linear 3-manifolds and that $f$ is a continuous mapping of $M^{3}$ onto $N^{3}$ such that $f^{-1}(y) \in U V^{\infty}$, for each $y \in N^{3}$. Then, for each $y \in N^{3}$, and for each open set $U \subset M^{3}$ containing $X=f^{-1}(y)$, there is an open set $V$ such that $X \subset V \subset U$ and $V-X$ is topologically $S^{2} \times(0,1)$.

Proof. Using the techniques of [7, Theorem 2.1], or [4, Lemma 5], or [2, Corollary 6.5$]$, we see that each set $f^{-1}(y)$ satisfies the cellularity criterion in $M^{3}$. The result follows by Theorem 1 .

COROLLARY 2.1. Under the hypotheses of Theorem 2, there are at most a finite number of $y \in N^{3}$ such that $f^{-1}(y)$ fails to be cellular in $M^{3}$. Further, $N^{3}$ can be obtained by removing a finite, disjoint collection of compact, polyhedral homotopy 3-cells from $M^{3}$ and replacing each by a piecewise-linear 3-cell.

Proof. The fact that only a finite number of inverse sets can fail to be cellular is immediate from Theorem 2, the compactness of $M^{3}$, and the fact that $\left\{f^{-1}(y) \mid y \in N^{3}\right\}$ is an upper semicontinuous decomposition of $M^{3}$ into compact sets. To prove the second assertion, let $y_{1}, \cdots, y_{n}$ be all those points $y$ of $N^{3}$ for which $f^{-1}(y)$ fails to be cellu- 
lar. Let $\pi$ be a mapping of $M^{3}$ onto a closed 3-manifold $K^{3}$ such that the only nondegenerate inverse sets of $\pi$ are $f^{-1}\left(y_{1}\right), \cdots, f^{-1}\left(y_{n}\right)$, and such that $K^{3}$ is (by Corollary 1.1) obtained from $M^{3}$ by a finite number of "surgeries" of the type described.

Let $g$ be defined so as to make the following diagram consistent:

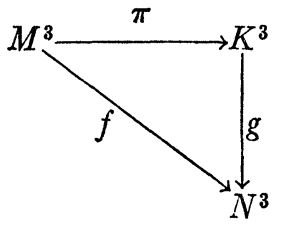

Then $g$ is a cellular mapping, and by [1], $N^{3}$ is homeomorphic to $K^{3}$, as required.

CoROLlary 2.2 Under the hypotheses of Theorem 2, if $M^{3}$ and $N^{3}$ are homeomorphic, then each set $f^{-1}(y)$ is cellular in $M^{3}$.

Proof. Reviewing the proof of Corollary 2.1, we see that it will suffice to show that if $F_{1}, \cdots, F_{n}$ is a finite disjoint collection of polyhedral, homotopy 3-cells in $M^{3}$, such that each $F_{i}$ fails to be a topological 3-cell, and if each $F_{i}$ is replaced by a (real) 3 -cell, then the resulting 3-manifold is not homeomorphic to $M^{3}$. If $M^{3}$ is an orientable 3-manifold, this is immediate from Milnor's "unique decomposition theorem" (Theorem 1 of [6]). If $M^{3}$ is nonorientable, we apply the same argument to the orientable double covering of $M^{3}$.

Corollary 2.3. Let $M^{3}$ and $N^{3}$ be closed, piecewise-linear 3-manifolds, and suppose that there exist continuous mappings

$$
f_{1}: M^{3} \rightarrow N^{3}, \quad f_{2}: N^{3} \rightarrow M^{3}
$$

of each of these 3-manifolds onto the other, such that $f_{1}^{-1}(y) \in U V^{\infty}$ and $f_{2}^{-1}(x) \in U V^{\infty}$, for each $x \in M^{3}, y \in N^{3}$. Then $M^{3}$ and $N^{3}$ are homeomorphic, and each point inverse of $f_{i}(i=1,2)$ is cellular.

Proof. This follows from Corollary 2.1 and Corollary 2.2 (and its proof).

\section{REFERENCES}

1. Steve Armentrout, Cellular decompositions of 3-manifolds that yield 3-manifolds, Abstract 634-53, Notices Amer. Math. Soc. 13 (1966), 374.

2. - Homotopy properties of decomposition spaces, (to appear).

3. H. Kneser, Geschlossen Flächen in dreidimensionalen Mannigfaltigkeiten, Jber. Deutsch. Math. Verein. 38 (1929), 248-260.

4. J. Martin, The sum of two crumpled cubes, Michigan Math. J. 13 (1966), 147151. 
5. D. R. McMillan, Jr., A criterion for cellularity in a manifold, Ann. of Math. 79 (1964), 327-337.

6. J. Milnor, A unique decomposition theorem for 3-manifolds, Amer. J. Math. 84 (1962), 1-7.

7. T. M. Price, $A$ necessary condition that a cellular upper semicontinuous decomposition of $E^{n}$ yield $E^{n}$, Trans. Amer. Math. Soc. 122 (1966), 427-435.

The University of Wisconsin

\section{THE $C^{*}$-ALGEBRA GENERATED BY AN ISOMETRY ${ }^{1}$}

BY L. A. COBURN

Communicated by P. R. Halmos, April 24, 1967

1. Introduction. In this paper, I determine the structure of any $C^{*}$-algebra generated by an isometry. Using a theorem of Halmos [3], the problem is reduced to the study of $C^{*}$-algebras $Q(A)$ generated by $A$ and $A^{*}$ where (i) $A$ is unitary, (ii) $A=S_{\alpha}$ with $S_{\alpha}$ the shift of multiplicity $\alpha$, and (iii) $A=W \oplus S_{\alpha}$ with $W$ unitary. In case (i), the resulting algebra is isometrically *-isomorphic to the algebra $C(\sigma(A))$ of all complex-valued continuous functions on the spectrum of $A$ and nothing more need be said. In cases (ii) and (iii), it turns out that $Q(A)$ is isometrically *-isomorphic to $Q\left(S_{1}\right)$ so that $Q(A)$ is independent of $W$ and $\alpha$. In each of these cases, there is a unique minimal closed two-sided ideal $\mathscr{g}(A)$ such that $Q(A) / \mathscr{G}(A)$ is isometrically *-isomorphic to $C(T)$, where $T$ is the perimeter of the unit circle. The ideal $\mathscr{g}(A)$ is determined spatially in the cases $A=S_{1}$ and $A=W \oplus S_{1}$.

We begin with the notation. For our purposes, all Hilbert spaces are complex and all ideals are closed and two-sided. If $\left\{e_{n}: n=0,1\right.$, $2, \cdots\}$ is an orthonormal basis for a Hilbert space $H$ then the shift $S=S_{1}$ is defined by $S e_{n}=e_{n+1}$. By a shift of multiplicity $\alpha$ is meant the $\alpha$-fold direct sum $S \oplus S \oplus \cdots \oplus S$ acting on $H \oplus H \oplus \cdots \oplus H$. The orthogonal projection onto the one-dimensional subspace of $H$ spanned by $e_{n}$ is denoted by $P_{n}$.

If $H\left(\right.$ or $\left.H_{i}\right)$ is a Hilbert space then $\beta(H)$ (or $B\left(H_{i}\right)$ ) denotes the algebra of all bounded operators with the usual norm topology and $\Re\left(\right.$ or $\mathcal{K}_{i}$ ) denotes the ideal of all compact operators. The natural quotient map from $B(H)$ to $B(H) / \mathscr{K}\left(B\left(H_{i}\right)\right.$ to $\left.B\left(H_{i}\right) / K_{i}\right)$ is given by

${ }^{1}$ Research supported by NSF Grant GP 5866. 